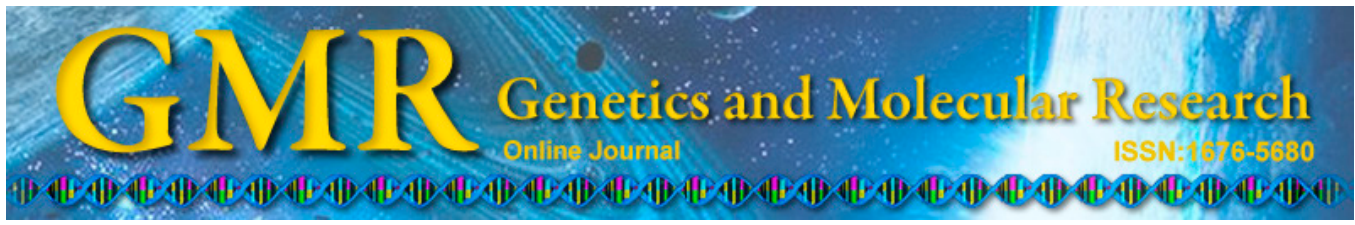

\title{
Swine leukocyte antigen class II genes (SLA- DRA, SLA-DRB1, SLA-DQA, SLA-DQB1) polymorphism and genotyping in Guizhou minipigs
}

Z.Z. Liu ${ }^{1 *}$, J.H. Xia ${ }^{1 *}$, L.L. Xin ${ }^{1}$, Z.G. Wang ${ }^{2}$, L. Qian ${ }^{3}$, S.G. Wu ${ }^{3}$, S.L. Yang ${ }^{1}$ and $\mathrm{K}$. $\mathrm{Li}^{1}$

${ }^{1}$ Institute of Animal Science, Chinese Academy of Agricultural Sciences, Beijing, China

${ }^{2}$ School of Life Science, Anhui Agricultural University, Anhui Hefei, China

${ }^{3}$ Guiyang College of Traditional Chinese Medicine, Guiyang, China

*These authors contributed equally to this study.

Corresponding author: S.L. Yang

E-mail: yangshulin@caas.cn

Genet. Mol. Res. 14 (4): 15256-15266 (2015)

Received June 21, 2015

Accepted September 19, 2015

Published November 30, 2015

DOI http://dx.doi.org/10.4238/2015.November.30.1

ABSTRACT. The swine leukocyte antigen (SLA) complex harbors
highly polymorphic gene clusters encoding glycoproteins that are
involved in responses to vaccines, infectious disease, and production
performance. Pigs with well-defined SLA class II genes are useful for the
study of disease, immunology, and vaccines. In this study, we analyzed
four SLA class II genes (SLA-DRA, SLA-DRB1, SLA-DQA, SLA-
DQB1) in 22 founder Guizhou minipigs using a sequence-based typing
method. Twelve alleles were detected, compared with the SLA class II
allele sequences in the GenBank, and one of twelve alleles was found
to be novel in Guizhou minipigs. There are four SLA II haplotypes, and
one of them has been previously reported in Meishan pigs. Furthermore,
based on sequence information of these alleles, we developed a simple 
SLA typing method implemented to SLA-typing for unknown offspring of Guizhou minipigs, relying on designed twelve sequence specific primers that could discriminate between each other. According to the combination of sequence-based typing and PCR-SSP, we were able to rapidly check SLA typing of Guizhou breeding stock and identified four SLA haplotypes in the herd. Therefore, SLA-defined Guizhou minipigs will be useful as animal models for xenotransplantation and immunological research.

Key words: SLA-DRA; SLA-DRB1; SLA-DQA; SLA-DQB1; Guizhou minipigs; PCR-site specific primer

\section{INTRODUCTION}

The swine leukocyte antigen (SLA) is a gene-dense region that contains a number of important immune-related genes, and includes class I, II, and III gene clusters located in the centromere spanning chromosome 7 . These SLA genes are highly polymorphic and are associated with disease resistance and economic traits (Shinkai et al., 2012). The study of SLA gene polymorphism is crucial to the research of immune responses (Essler et al., 2013).

Many SLA alleles and haplotypes from different pig herds worldwide such as large white pigs, Duroc pigs, and Meishan pigs have been analyzed, and are systematically named by the SLA Nomenclature Committee of the International Society for Animal Genetics (ISAG) and available in the Immune Polymorphism Database. Clearly, accurate SLA typing methods are crucial for inbreeding or selection of special haplotypes in immune response studies. Based on the polymerase chain reaction (PCR), there are a number of convenient and fast molecular biology typing methods, such as PCR-SSP (Ho et al., 2010) and PCR-RFLP (Lee et al., 2005). Sequence-based typing and PCR-sequence-specific primers (PCR-SSP) are wildly used for SLA typing, although this is only suitable for characterizing founder pigs due to labor-intensive and time-consuming experimental procedures to resolve heterozygous sequences (Smith et al., 2005). PCR-SSP has also been implemented extensively in human transplant-related tissue typing due to its high efficiency, accuracy, and reproducibility (Ho et al., 2009b).

SLA class II genes play a critical role in the immune response to foreign antigens and to vaccines. These genes are also involved in the differentiation of myeloid dendritic cells from plasmacytoid or natural interferon producing cells (Summerfield et al., 2003). SLA class II genes also control infectious disease responses and influence vaccine specificity and efficacy (Lopez Fuertes et al., 1999). The matching of SLA class II is required for acceptance of solid organ allografts and bone marrow cell transplants (Sumitran-Holgersson et al., 2003). Repression or expression of SLA class II antigens should permit the study of transplant rejection and tolerance well (Quinn et al., 2003). The Guizhou minipig is a precious species, which is maintained as a closed colony in Guizhou Province in China. This valuable resource pig is an excellent laboratory animal because of its early sexual maturation, small body size, and pure genetic background (Yang et al., 2007). There have been reports associated with SLA of this herd (Chen et al., 2003); however, this study was not performed in a systematic manner and only involved the sequences of one gene or exon. Therefore, further studies on Guizhou minipig SLA genes for use in xenotransplantation and immune research are required. In this study, four SLA class II genes (DRA, DRB1, DQA, DQB1) of 22 Guizhou minipig founders 
were sequenced and a SLA class II genotyping assay was developed based on the PCR sequence specific primers for specific haplotypes.

\section{MATERIAL AND METHODS}

\section{Animals}

The closed colony of Guizhou minipig was founded by the Guiyang College of Traditional Chinese Medicine in 1982. Initially, four sows and two boars were obtained from the original location of the Guizhou Xiang pigs and bred under closed conditions. The Germplasm Resource Center of Chinese Laboratory Minipigs was established in 2004 in the Institute of Animal Science, CAAS, supported by the Ministry of Science and Technology of China. Twenty-two individuals derived from the $19^{\text {th }}$ generation of the Guizhou minipigs colony and were introduced to the center for Chinese minipig germplasm resource conservation in 2006. Ear samples from 22 individuals and their five descendants were obtained and preserved at $-80^{\circ} \mathrm{C}$.

\section{RNA extraction and PCR amplification}

Ear samples from Guizhou minipigs were used to extract total RNA, using an RNA extraction kit (BioTeke, Peking, China), and cDNA was obtained using a first strand cDNA synthesis kit (Fermentas Life Science, Peking, China ) according to manufacturer instructions. In order to amplify the entire coding region, locus-specific primers of SLA class II genes (SLA-DRA, SLA-DRB1, SLA-DQA, and SLA-DQB1) were designed against the untranslated regions (Table 1). Several of these primers have been previously described. Four primer pairs of SLA class II genes were referred to in a study by Smith et al. (2005). The cDNA was amplified using LA Taq with GC buffer (Takara, Peking, China) in SLA class II genes (SLADRA, SLA-DRB1, SLA-DQA, and SLA-DQB1). Amplification was conducted as follows, 5 min at $94^{\circ} \mathrm{C}, 35$ cycles of $94^{\circ} \mathrm{C}$ for $30 \mathrm{~s}$, each specific annealing temperature for $30 \mathrm{~s}$ and $72^{\circ} \mathrm{C}$ for $120 \mathrm{~s}$, and extension at $72^{\circ} \mathrm{C}$ for $5 \mathrm{~min}$, and thermal cycling was performed in an Eastwin Life Sciences EDC-810 thermal cycler (Eastwin Life Sciences, Peking, China). Sequences of SLA loci primers and their annealing temperatures are shown in Table 1.

\begin{tabular}{|c|c|c|c|c|}
\hline Locus & Forward/Reverse primer $\left(5^{\prime}-3^{\prime}\right)$ & Annealing temp $\left({ }^{\circ} \mathrm{C}\right)$ & Product (bp) & Reference \\
\hline SLA-DRA & $\begin{array}{l}\text { F: CATCGAGGCATCTAAGGAGA } \\
\text { R: CAAAGTCCATTCCCTGCAAG }\end{array}$ & 55 & 805 & Smith et al., 2005 \\
\hline SLA-DRB1 & $\begin{array}{l}\text { F: TGTCCTCTCCTGTTCTCCA } \\
\text { R: AGGACGCAGAGCATAGCAG }\end{array}$ & 52 & 907 & Smith et al., 2005 \\
\hline SLA-DQA & $\begin{array}{l}\text { F: AACTCCGAAGAGCAACAGC } \\
\text { R: ACCTTCCCTTCTGGAGTGTG }\end{array}$ & 55 & 800 & Smith et al., 2005 \\
\hline SLA-DQB1 & $\begin{array}{l}\text { F: TGACTACCATTACTTCTTCGT } \\
\text { R: TCTTGCACAGTCTGTTGAGG }\end{array}$ & 54 & 1103 & Smith et al., 2005 \\
\hline
\end{tabular}

\section{Gene cloning and sequencing}

The purified PCR products were cloned into a pMD18-T cloning vector (Takara, Da- 
lian, China), according to manufacturer instructions. In order to identify the alleles of each locus, eight positive clones per locus were sequenced bidirectional in individual and additional clones and were sequenced to resolve discrepancies. Sequencing reactions were performed (Invitrogen, Beijing, China). Cloned inserts were sequenced in both directions using two universal primers (M13-47: 5'-CGCCAGGGTTTTCCCAGTCACGAC-3' and RV-M: 5'-GAGCGGATAACAATTTCACACAGG-3'). The SeqMan software in DNASTER version 7.1.0 was used to analyze sequences. The sequences of RT-PCR products of each gene were assembled by over lapping forward and reverse sequences. To accurately confirm an allele, it must be sequenced in more than two individuals or obtained in one individual repeatedly. The false sequences were excluded through comparison between cloned sequences and the peaks observed in sequencing profiles. The coding sequence of SLA class II alleles were assembled and compared with sequences deposited in the GenBank database, and novel sequences were submitted to the IPD-MHC and GenBank sequence database.

\section{Phylogenetic analysis of Swine leukocyte antigen class II genes}

Phylogenetic trees of SLA genes were constructed by the neighbor-joining method (Saitou, 1987) with Kimura 2-parameters (Kimura, 1980) using the MEGA4 program (Tamura et al., 2007). Every tree included all SLA nucleotide sequences obtained from each locus in this study, together with SLA nucleotide sequences from the IPD-MHC Database of swine (http://www.ebi.ac.uk/ipd/mhc/download.html). Bootstrap values were obtained based on 1000 replicates.

\section{Alleles sequence specific primer and PCR-SSP}

Twelve site-specific primers were designed (Primer Premier, version 5.00) from sequences of Guizhou founder minipigs, which could distinguish different alleles in this closed colony (Table 2). The designation of allele-specific primer sets were conducted with the principle that the 3 ' end of one or both primers covered polymorphic sites unique to their corresponding allele, and that each allele-specific primer pair was located in the same exon, generally in exon 2 or exon 3, which showed high polymorphism (Lunney et al., 2009). Two SLA-DRA, two SLA-DRB1, four SLA-DQA, and four SLA-DQB1 primer pairs were designed. Pig genomic DNA was extracted from ear tissue using the TIANamp Genomic DNA kit (TIANGEN, Peking, China), and was used in SLA-typing using the PCR-SSP method. Touch-down PCR was used (Yeom et al., 2010) to minimize unspecific amplification. This consisted of incubation at $95^{\circ} \mathrm{C}$ for $1 \mathrm{~min}, 5$ cycles of $95^{\circ} \mathrm{C}$ for $30 \mathrm{~s}, 68^{\circ} \mathrm{C}$ for $30 \mathrm{~s}, 72^{\circ} \mathrm{C}$ for $30 \mathrm{~s}, 5$ cycles of $95^{\circ} \mathrm{C}$ for $30 \mathrm{~s}, 63^{\circ} \mathrm{C}$ for $30 \mathrm{~s}, 72^{\circ} \mathrm{C}$ for $30 \mathrm{~s}$, and 30 cycles of $95^{\circ} \mathrm{C}$ for $30 \mathrm{~s}$, annealing temperature (Table 2) for $30 \mathrm{~s}, 72^{\circ} \mathrm{C}$ for $30 \mathrm{~s}$, and a final incubation of $72^{\circ} \mathrm{C}$ for 5 min. Thermal cycling was performed in an Eastwin Life Sciences EDC-810 Thermal Cycler (Eastwin Life Sciences, Peking, China).

\section{RESULTS}

\section{SLA class II gene alleles and haplotypes of Guizhou minipigs}

The SLA class II alleles of the Guizhou minipigs were analyzed by mRNA sequence- 


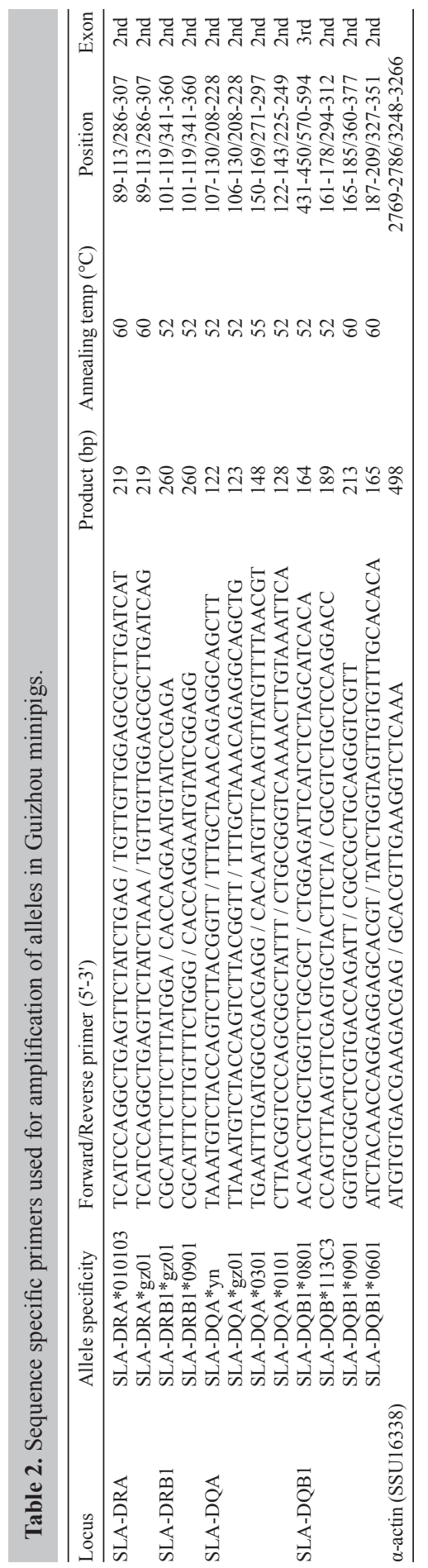


based SLA typing in samples from 22 individuals, and more than 704 clones were sequenced from four loci of the founder pigs. Twelve alleles from Guizhou minipigs were identified in the four SLA class II loci, their distribution is two SLA-DRA alleles, two SLA-DRB1 alleles, four SLA-DQA alleles, and four SLA-DQB1 alleles (Table 3). There was one novel SLA-DRA allele in the closed colony, when compared with sequences in the GenBank database, in addition the complete coding sequence and protein sequence had been deduced and submitted to the GenBank (Table 3). The novel SLA-DRA allele was given a temporary name: SLA-DRA*gz01 (accession: JQ361659) in 17 seventeen individuals. The other 11 alleles were previously reported, and the official names given by ISAG are: SLA-B*m (accession: AF464049.2), SLA-DRA*010103 (accession: EU432070.1), SLA-DRB1*0901 (accession: EU432073.1), SLA-DQA*yn (accession: AY102475.1), SLA-DQA*0301 (accession: EU722913.1), SLA-DQA*0101 (accession: DQ883218.1), SLA-DQB1*0801 (accession: DQ303219.1), SLA-DQB1*113C3 (accession: AY135574.1), SLA-DQB1*0901 (accession: EU432063.1), SLA-DQB1*0601 (accession: DQ883219.1), and one SLA-DRB1 allele (accession: AK347078.1), one SLA-DQA allele (accession: AK230483.1) existed in the GenBank but the name was absent, for the sake of convenience, this was temporarily named as SLA-DRB1*gz01 (accession: AK347078.1) and SLA-DQA*gz01 (accession: AK230483.1).

\begin{tabular}{|c|c|c|c|c|c|}
\hline \multirow[t]{2}{*}{ ID } & \multicolumn{4}{|c|}{ CLASS II } & \multirow[t]{2}{*}{ Haplotype } \\
\hline & SLA-DRA & SLA-DRB1 & SLA-DQA & SLA-DQB1 & \\
\hline G100 & 010103 & 0901 & 0301 & 0801 & Hp-gz00.14 \\
\hline G118 & 010103 & 0901 & 0301 & 0801 & Hp-gz00.14 \\
\hline G140 & 010103 & 0901 & 0301 & 0801 & Hp-gz00.14 \\
\hline G151 & 010103 & 0901 & 0301 & 0801 & Hp-gz00.14 \\
\hline G122 & 010103/gz01 & 0901/gz01 & 0101/0301 & 0601/0801 & Hp-gz00.00 \\
\hline G106 & gz01 & gz01 & 0101 & 0601 & Hp-gz00.01 \\
\hline G108 & gz01 & gz01 & 0101 & 0601 & Hp-gz00.01 \\
\hline G114 & gz01 & gz01 & 0101 & 0601 & Hp-gz00.01 \\
\hline G338 & gz01 & gz01 & 0101 & 0601 & Hp-gz00.01 \\
\hline G398 & gz01 & gz01 & 0101 & 0601 & Hp-gz00.01 \\
\hline G126 & gz01 & gz01 & 0101 & 0601 & Hp-gz00.01 \\
\hline G104 & 010103/gz01 & 0901 & 0301 & 0601/0801 & \\
\hline G107 & 010103/gz01 & 0901/gz01 & 0101/0301 & 0601/0801 & Hp-gz00.00 \\
\hline G125 & 010103/gz01 & 0901 & yn/gz01 & $113 \mathrm{C} 3 / 0901$ & \\
\hline G130 & gz01 & 0901/gz01 & $0101 / 0301$ & 0601/0801 & \\
\hline G302 & $010103 / \mathrm{gz} 01$ & 0901 & 0101/0301 & 0601/0801 & \\
\hline G322 & 010103/gz01 & 0901/gz01 & 0101/0301 & 0601/0801 & Hp-gz00.00 \\
\hline G394 & gz01 & gz01 & 0101/gz01 & 0601 & \\
\hline G135 & 010103 & 0901 & 0101/0301 & 0801 & \\
\hline G310 & 010103/gz01 & 0901/gz01 & 0101/0301 & 0601 & \\
\hline G6 & 010103/gz01 & 0901/gz01 & $\mathrm{yn} / \mathrm{gz} 01$ & $113 \mathrm{C} 3 / 0901$ & \\
\hline G396 & 010103/gz01 & 0901/gz01 & gz01113C3/0901 & & \\
\hline
\end{tabular}

Because of the strong linkage disequilibrium of the SLA loci, the haplotype nomenclature system was established by the SLA Nomenclature Committee of ISAG (Ho et al., 2009a). High-resolution SLA haplotypes are named with the prefix "Hp-", and a number for the class I haplotype followed by a number for class II haplotype separated by a period (e.g. Hp-1.1). The results of the nucleotide sequence analysis of RT-PCR products in the SLA class II genes, two class II haplotypes were obtained from the 22 founder Guizhou minipigs (Table 3). One novel class II haplotype was temporarily named as Hp-gz00.01 (SLA-DRA*gz01, 
SLA-DRB1*gz01, SLA-DQA*0101, SLA-DQB1*0601) and was confirmed in six pigs (G106, G108, G114, G338, G398, and G126). Another SLA class II haplotype that has been previously published and is present in Meishan pigs (Ho et al., 2006), and was officially named as Hp-00.14 (SLA-DRA*010103, SLA-DRB1*0901, SLA-DQA*0301, SLA-DQB1*0801), was confirmed in four pigs (G100, G118, G140, and G151). Crossover of class II haplotypes was observed in the G122 individual, and the crossover occurring between the Hp-gz0.14 and Hp-gz0.01 haplotypes, resulted in new recombinant haplotypes (SLA-DRA*gz01, SLADRB1*gz01, SLA-DQA*0101, SLA-DQB1*0601). A crossover that occurred between SLADRA and SLA-DRB1 loci was observed in G130, whose SLA II haplotypes may come from recombination between Hp-gz0.01 and Hp-gz0.14, resulting in a new SLA II recombinant haplotype (SLA-DRA*gz01, SLA-DRB1*0901, SLA-DQA*0301, SLA-DQB1*0801).

\section{Phylogenetic analysis of alleles from the SLA class II gene}

To investigate the genetic relationship between the identified novel SLA-DRA alleles and all previously reported alleles of each locus, phylogenetic trees were constructed using the neighbor-joining method (Saitou, 1987). In the SLA-DRA phylogenetic tree, the SLA-DRA*gz01 allele is more closely related to SLA-DRA*010101. However, as the highly conservative and low polymorphism features in SLA-DRA genes, closely phylogenetic relationship among them was shown on the tree, and the scale of tree was change to 0.0005 presenting (Figure 1).

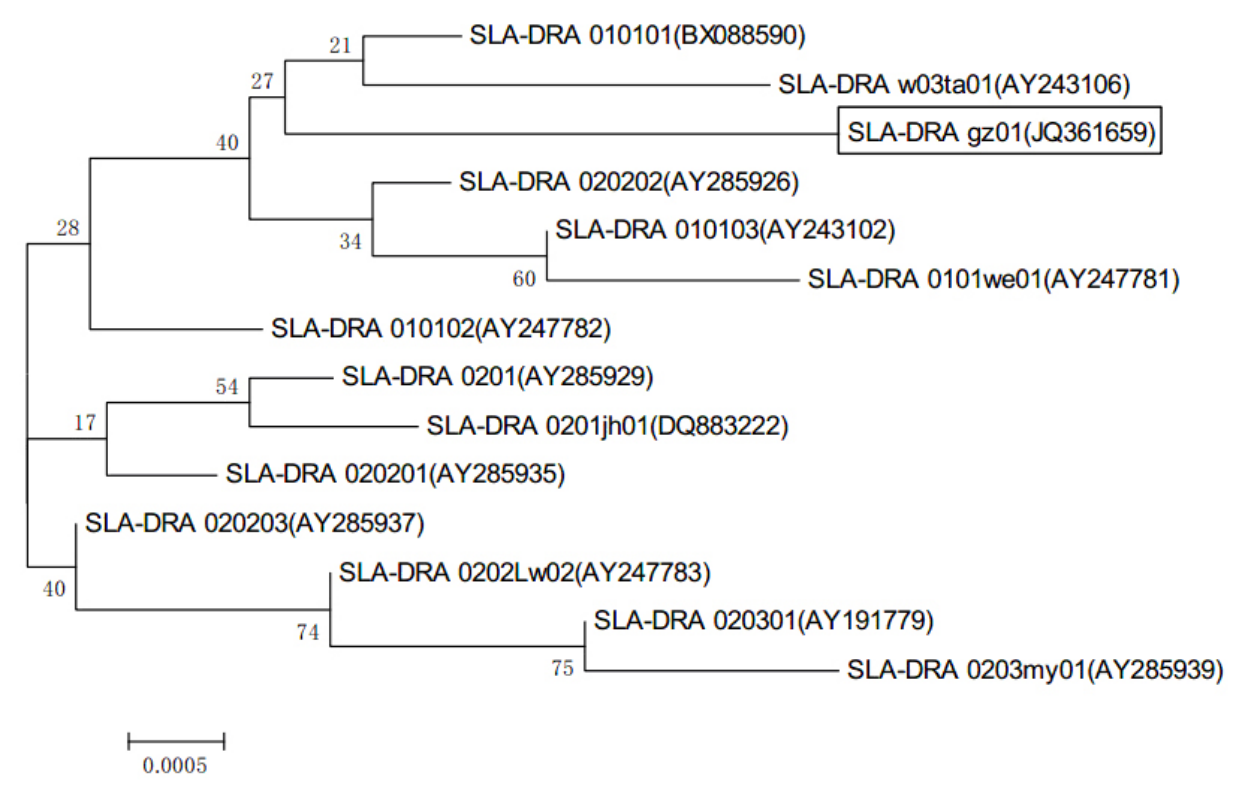

Figure 1. Phylogenetic trees of SLA class II loci alleles in the IPD-MHC database and novel alleles identified in Guizhou minipigs. The complete ORF nucleotide sequence alleles were analyzed using the neighbor-joining method on the basis of Kimura 2-parameter distances using the MEGA4 program. A box indicates a novel SLA allele identified in this study. 


\section{SLA-typing by PCR-SSP in Guizhou minipigs}

In order to distinguish each of the alleles found in Guizhou minipigs, sequencespecific primers were designed, according to the mismatch at the $3^{\prime}$ end of the primer as previously described (Cho et al., 2010). The PCR-SSP was conducted with sequenced allele clones as templates to validate the specificity of each allele SSP, and the lack of nonspecific amplification was confirmed. After confirming the sensitivity of SSP, PCR-SSP SLA class II gene typing was carried out using genomic DNA as a template. The results were mostly consistent with mRNA sequence-based SLA typing. Although eight clones were sequenced for each locus, occasionally only one allele was detected when two alleles should be present at heterozygous loci. Fortunately, PCR-SSP SLA typing enabled us to locate the missed alleles: SLA-DRA*010103 in two pigs (G6 and G396), SLA-DRB1*gz01 in one pig (G107).

We conducted PCR-SSP for five additional descendants in the founder Guizhou minipigs (Figure 2). Each PCR-SSP reaction was multiplexed into an internal control (Ho et al., 2006), which amplifies a portion of the $\alpha$-actin gene that is visible as a band at $498 \mathrm{bp}$. We found two SLA class II homozygote pigs (G423, G460, G465), which had the same SLA haplotype alleles (Hp-gz0.14, Hp-gz0.01) as their mothers (G100, G108) respectively. Another two (G425, G489) were class II heterozygote pigs (Table 4).

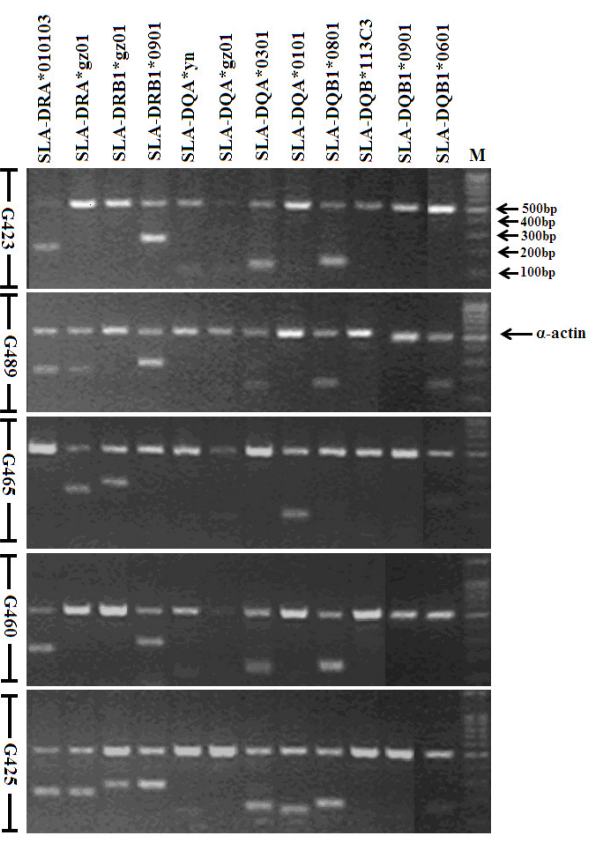

Figure 2. Example of SLA genotyping of Guizhou minipigs at four loci (SLA class II: SLA-DRA, SLA-DRB1, SLA-DQA, SLA-DQB1) by PCR-SSP using the full primer set, 12 primer pairs, specific for each alleles of the herd in Table 2. Positive control primers, which amplify a portion of the porcine $\alpha$-actin gene ( $498 \mathrm{bp}$ ), were multiplexed into each reaction to check for adequate amplification. Allele-specific primers were designed to amplify PCR products smaller than the positive control. The presence of a band in a given lane indicates that the pig is positive for that allele. Product sizes of each allele-specific primer are given in Table 2. If the positive control or the allele specific band is present, the reaction would be scored. Lanes from left to right represent 12 different genes. G423, G425, G460, G465, and G489 represent five Guizhou minipigs descendants. Lane $M=100$-bp PCR marker. 


Table 4. Additional PCR-SSP SLA typing for five descendants of Guizhou minipigs.
\begin{tabular}{lccccc}
\hline \multicolumn{7}{l}{ ID } & \multicolumn{3}{c}{ CLASS II } & Haplotype \\
\cline { 2 - 5 } & SLA-DRA & SLA-DRB1 & SLA-DQA & SLA-DQB1 & \\
\hline G423 & 010103 & 0901 & 0301 & 0801 & Hp-gz0.14 \\
G425 & $010103 / \mathrm{gz} 01$ & 0901 & 0301 & $0601 / 0801$ & Hp-gz0.00 \\
G460 & gz01 & gz01 & 0101 & 0601 & Hp-gz0.01 \\
G465 & 010103 & 0901 & 0301 & 0801 & Hp-gz0.14 \\
G489 & $010103 /$ gz01 & gz01/0901 & $0101 / 0301$ & $0601 / 0801$ & \\
\hline
\end{tabular}

\section{DISCUSSION}

The SLA class II gene complex plays a critical role during vaccination, infectious disease, and production performance. In this study, we employed a combinational method with mRNA sequence-based and PCR-SSP typing to identify SLA class II polymorphism in Guizhou minipigs as previously described (Ho et al., 2010). Because of the lack of human organ and the similar physiological characteristics between human and porcine, more attention is given to organ grafts of pigs in the study of xenotransplantation. Pigs with SLA haplotypes that are homozygous for SLA loci are invaluable for the study of immune responses to allo- or xenografts (Yeom et al., 2010). Therefore, this study may contribute to the selection of xenotransplantation pigs.

Guizhou minipigs are a precious rare species of China, and we are therefore aiming to develop a practical SLA typing method. Although DNA sequencing is the most precise method of SLA typing, it is also expensive and labor intensive, while simple and fast, the PCR-SSP method is only suited to already known sequences. Allelic dropouts occur occasionally when using sequence-based typing methods due to preferential PCR amplification of one allele compared to another in a heterozygous sample (Ho et al., 2009a), conversely, PCR-SSP can identify these lost alleles. Combination of the two methods can be effectively applied to outbred pig populations. In order to eliminate some PCR- or cloning-generated artificial sequences, we adopted the following criteria: sequences were considered to be true alleles only when occurring in more than one clone, from the same or from different individuals, and when they were confirmed by two independent PCR runs (Gao et al., 2014).

SLA class II genes at four loci of 22 founder Guizhou minipigs were characterized, and a PCR-SSP typing method was established. Finally, a total of 12 alleles and two class II haplotypes were identified, and one allele and one haplotype were found to be novel. By comparing the results in BLAST, the novel SLA-DRA*gz01 was found to be more similar to the SLA-DRA*010101 allele (accession: DQ883224.1), which was also found by Lee et al. (2008) in a native breed of Korean pig, between which there were only three base mismatches (position: 111, 113, 286) on the second exon. Another 11 alleles and one SLA class II haplotype (Hp-00.14) matched with Meishan pigs were already documented in the IPD database. SLA-DRB1*0901 (accession: EU432073.1) of Hp-00.14 presented in Hp-00.11 is published in Sinclair pigs, SLA-DRA*010103 (accession: EU432070.1) was documented in Hp-00.18 of Meishan pigs. SLA-DQA*0101 (accession: DQ883218.1) and SLA-DQA*0301 (accession: EU722913.1) were included in Hp-00.01 of Large White and Korean native pigs and Hp-00.12 of Sinclair pigs respectively, SLA-DQB1*0601 (accession: DQ883219.1) was in Hp-00.07 of Yucatan pigs as well as Hp-00.16 of Clawn pigs, and SLA-DQB1*0801 (ac- 
cession: DQ303219.1) appears in two haplotypes, Hp-00.06 of Yucatan pigs and Hp-00.10 of Sinclair and Hanford pigs. In some studies, the SLA-DQA and SLA-DRA loci were not characterized due to tight linkage to the SLA-DQB1 and SLA-DRB1 loci and the limited polymorphism, while in our study, we detected four alleles in the SLA-DQA loci and two in the SLA-DRA loci. Linkage between SLA genes is not always static, and that crossover and sequence variation can occur. Therefore, to avoid losing alleles, it would be best to take account of these two important loci even when characterizing the unclear genetic background of outbred populations.

In conclusion, this study makes three contributions to xenotransplantation research. First, we analyzed another herd of minipigs with well-characterized SLA class II antigens and available homozygous lines. Second, one novel SLA class II allele and one novel haplotype were discovered that could enrich the IPD-MHC database. Third, we establish a method that can be used to characterize the SLA types of this experimental herd of Guizhou minipigs. Sequence-based typing can be performed on the founder or parental pigs, and then allele specific PCR primers can be designed to type any offspring using PCR with site specific primers (PCRSSP). This method can also be used to establish a new SLA homozygote line.

\section{ACKNOWLEDGMENTS}

Research supported by a fund from the National Key Technology Support Program (\#2012BAI39B04), National Natural Science Foundation of China (\#31372276), Agricultural Science and Technology Innovation Program (\#ASTIP-IAS05), National Basic Research Program (\#2011CBA01005), National Science and Technology Major Project (\#2013ZX08010003) and Development Program of China (\#SQ2011AAJY2795).

\section{REFERENCES}

Cho HO, Ho CS, Lee YJ, Cho IC, et al. (2010). Establishment of a resource population of SLA haplotype-defined Korean native pigs. Mol. Cells 29: 493-499.

Chen FX, Tang J, Li NL, Shen BH, et al. (2003). Novel SLA class I alleles of Chinese pig strains and their significance in xenotransplantation. Cell Res. 13: 285-294.

Essler SE, Ertl W, Deutsch J, Ruetgen BC, et al. (2013). Molecular characterization of swine leukocyte antigen gene diversity in purebred Pietrain pigs. Anim. Genet. 44: 202-205.

Gao C, Jiang Q, Guo D, Liu J, et al. (2014). Characterization of swine leukocyte antigen (SLA) polymorphism by sequencebased and PCR-SSP methods in Chinese Bama miniature pigs. Dev. Comp. Immunol. 45: 87-96.

Ho CS, Rochelle ES, Martens GW, Schook LB, et al. (2006). Characterization of swine leukocyte antigen polymorphism by sequence-based and PCR-SSP methods in Meishan pigs. Immunogenetics 58: 873-882.

Ho CS, Franzo-Romain MH, Lee YJ, Lee JH, et al. (2009a). Sequence-based characterization of swine leucocyte antigen alleles in commercially available porcine cell lines. Int. J. Immunogenet. 36: 231-234.

Ho CS, Lunney JK, Franzo-Romain MH, Martens GW, et al. (2009b). Molecular characterization of swine leucocyte antigen class I genes in outbred pig populations. Anim. Genet. 40: 468-478.

Ho CS, Martens GW, Amoss Jr MS, Gomez-Raya L et al. (2010). Swine leukocyte antigen (SLA) diversity in Sinclair and Hanford swine. Dev. Comp. Immunol. 34: 250-257.

Kimura M (1980). A simple method for estimating evolutionary rates of base substitutions through comparative studies of nucleotide sequences. J. Mol. Evol. 16: 111-120.

Lee JH, Simond D, Hawthorne WJ, Walters SN, et al. (2005). Characterization of the swine major histocompatibility complex alleles at eight loci in Westran pigs. Xenotransplantation 12: 303-307.

Lee YJ, Cho KH, Kim MJ, Smith DM, et al. (2008). Sequence-based characterization of the eight SLA loci in Korean native pigs. Int. J. Immunogenet. 35: 333-334.

Lopez Fuertes L, Domenech N, Alvarez B, Ezquerra A, et al. (1999). Analysis of cellular immune response in pigs 
recovered from porcine respiratory and reproductive syndrome infection. Virus Res. 64: 33-42.

Lunney J, Ho C, Wysocki M, Smith D (2009). Molecular genetics of the swine major histocompatibility complex, the SLA complex. Dev. Comp. Immunol. 33: 362-374.

Quinn G, Bower R, Dos-Santos Cruz G, Giovino M, et al. (2003). Structural and functional characteristics of a dominantnegative isoform of porcine MHC class II transactivator. Eur. J. Immunogenet. 30: 259-270.

Saitou NNM (1987). The neighbor-joining method: A new method for reconstructing phylogenetic trees. Mol. Biol. Evol. 4: 406-425.

Shinkai H, Arakawa A, Tanaka-Matsuda M, Ide-Okumura H, et al. (2012). Genetic variability in swine leukocyte antigen class II and Toll-like receptors affects immune responses to vaccination for bacterial infections in pigs. Comp. Immunol. Microbiol. Infect Dis. 35: 523-532.

Smith DM, Martens GW, Ho CS, Asbury JM (2005). DNA sequence based typing of swine leukocyte antigens in Yucatan Miniature Pigs. Xenotransplantation 12: 481-488.

Summerfield A, Guzylack-Piriou L, Schaub A, Carrasco CP, et al. (2003). Porcine peripheral blood dendritic cells and natural interferon-producing cells. Immunology 110: 440-449.

Sumitran-Holgersson S, Brevig T, Widner H and Holgersson J (2003). Activated porcine embryonic brain endothelial cells induce a proliferative human T-lymphocyte response. Cell Transplant. 12: 637-646.

Tamura K, Dudley J, Nei M and Kumar S (2007). MEGA4: molecular evolutionary genetics analysis (MEGA) software version 4.0. Mol. Biol. Evol. 24: 1596-1599.

Yang SL, Wang H, Feng ST and Wang A (2007). Investigation of the blood biochemistry parameters of three Chinese indigenous laboratory miniature pig breeds. China Anim. Husbandry Vet. Med. 34: 75-78.

Yeom SC, Park CG, Lee BC and Lee WJ (2010). SLA typing using the PCR-SSP method and establishment of the SLA homozygote line in pedigreed SNU miniature pigs. Anim. Sci. J. 81: 158-164. 